\title{
INFORMACIÓN BIBLIOGRÁFICA
}



ISSN: $1130-2887$

Gerardo Aboy CARLes. Las dos fronteras de la democracia argentina. La reformulación de las identidades de Alfonsin a Menem. Rosario, Santa Fe: HomoSapiens Ediciones, 2001. 333 pp.

Las dos fronteras de la democracia argentina. La reformulación de las identidades políticas de Alfonsín a Menem de Gerardo Aboy Carlés analiza el proceso político argentino abierto con la recuperación democrática desde la perspectiva de las identidades políticas. Este libro, publicado por la editorial argentina HomoSapiens -como parte de la colección Politeia, dirigida por Hugo Quiroga- es el resultado final y depurado de la tesis de doctorado en Ciencia Política y Sociología del autor en el marco del Instituto Ortega y Gasset y la Universidad Complutense de Madrid.

Este libro comienza con un profundo e interesante recorrido teórico, desde una visión multidiscipliar -que incorpora aportes de la sociología y la teoría política, la filosofía, la antropología, la semiología, la historia, la lingüística y la psicología- del concepto de identidad política. A partir de allí, Aboy Carles establece una serie de dimensiones analíticas constitutivas del concepto, que critica, discute y reformula para crear una definición operativa que le permita el análisis particular del proceso político argentino en las últimas dos décadas. Estas dimensiones -la representación, la alteridad y la tradición-, como bien señala el autor en la breve presentación, son «de especial utilidad para abordar el estudio de los procesos de constitución de unidades gregarias de acción política que alcanzan cierta estabilidad y permanencia». Allí es donde Aboy Carlés centra el objetivo de su trabajo; en el cuestionamiento de las continuidades y cambios producidos en las principales identidades políticas argentinas posteriores a la recuperación de la institucionalidad democrática en 1983.

Para dar paso al examen concreto de su objeto de análisis (y abordar así el estudio de los actores políticos y sociales que caracterizan a la sociedad argentina actual), primeramente, delimita teóricamente un concepto a todas luces escurridizo y complejo. Esta delimitación analítica es producto de un diálogo constante a través de todo el capítulo 1 con autores como Rousseau, Marr, Cerroni, Gramsci, Bourdieu, Schmitt, Žižek, por nombrar sólo algunos, y principalmente con Ernesto Laclau y su obra, que le sirven a Aboy Carlés como sustento para construir una reinterpretación conceptual de la identidad política. En este punto, hay que subrayar que el libro satisface la condición necesaria de toda tesis doctoral al plantear, a partir de la correspondiente revisión teórica, una nueva categoría que permite repensar los procesos político-sociales actuales a la luz de marcos diferenciales que aportan novedad y claridad al objeto de estudio. 
Una vez definido el concepto de identidad política, Aboy Carlés se interna de lleno en la realidad argentina. El segundo capítulo, «La formación política argentina previa a 1983» explora los procesos de construcción de las principales identidades políticas, la radical y la peronista, y su vinculación con la ampliación del sistema político. A partir de un recorrido histórico analítico, en el que aparecen rasgos diferenciales de la evolución de las dos fuerzas políticas argentinas más importantes, principalmente a partir de mediados del siglo XX -caracterizados sobre todo por la relación conflictiva que ambas establecen entre sí-, el autor se detiene principalmente en los elementos comunes que signaron las experiencias del radicalismo yrigoyenista y del peronismo. Como aclara Aboy Carlés en la presentación, se busca profundizar en «el estudio de la sedimentación de elementos disruptivos para el establecimiento de un régimen político democrático estable»; elementos que el autor considera estrechamente vinculados a la forma en que ambas identidades lograron constituirse como sucesivos procesos de ciudadanización que supusieron, por otra parte, una difícil coexistencia entre democracia y liberalismo.

Este segundo capítulo centra su atención en la relación establecida en la constitución de las identidades políticas previas a 1983 con la ampliación del sistema político (la experiencia yrigoyenista que derivó en la universalización del sufragio masculino nativo o naturalizado y la extensión de éste a las mujeres por el peronismo, la incorporación de nuevos sectores sociales a la política, etc.) como un único proceso.

El capítulo 3 «La ruptura alfonsinista» comienza con la delimitación del concepto de «frontera política». Tanto el peronismo como el radicalismo, a partir del proceso de redemocratización, reformulan sus identidades políticas proponiendo, especialmente, la ruptura respecto a la formación política previa. Aboy Carlés analiza cómo a partir de la recuperación democrática argentina, el alfonsinismo intenta quebrar con ciertos hábitos y rutinas sedimentadas; examina los ejes básicos a partir de los cuales emerge un discurso de quiebre respecto del pasado y profundiza en la mutación que sufre la política argentina (en todas las áreas: política exterior, sindical, judicial, entre otras) como causa del nuevo radicalismo planteado por Alfonsín.

El mismo presupuesto de análisis se plantea para el menemismo en el capítulo 4 «De Malvinas al menemismo, renovación y contrarrenovación en el peronismo». El autor estudia la ruptura central que supuso en la identidad peronista, la elección por uno de los componentes tradicionales del discurso de este partido. Plantea la pérdida de la dimensión populista en el menemismo como elemento clave para comprender la transformación de los elementos básicos de la antigua identidad peronista. El carácter exploratorio de este capítulo tiene la pretensión de establecer ciertas hipótesis vinculadas con la relación peronismo-menemismo, centrando el análisis en un aspecto polémico: la continuidad o ruptura con la identidad populista.

A lo largo de las páginas que componen este libro, el lector se encontrará con un impecable ejercicio intelectual por parte del autor en el que se combinan de manera equilibrada una justa dosis de reflexión teórica y un fructífero acercamiento a la realidad argentina. La reconstrucción del proceso histórico-político visto desde la mirada 
de las identidades políticas de sus principales actores -su construcción, sus giros, sus rupturas- es, en este sentido, la recuperación por el interés en un tema fundamental que había quedado opacado por el desarrollo de otros estudios vinculados principalmente a las transiciones y consolidaciones democráticas de los países latinoamericanos.

Los méritos de este trabajo no son otros que recuperar una visión que, tanto la sociología política como la ciencia política, habían marginado en pos de otros análisis pero que deviene imprescindible para comprender la historia, la política y la sociedad argentina. Estudiantes, docentes y especialistas de diferentes disciplinas encontrarán en ella una fuente sumamente útil para la comprensión de la realidad argentina actual y un sobresaliente trabajo capaz de combinar de manera seria y exhaustiva la utilización de los modernos instrumentos del análisis político aplicado a los acontecimientos recientes de la historia argentina.

PATRICIA MARENGHI

Jorge LANZARo (coord.). Tipos de presidencialismo y coaliciones políticas en América Latina. Buenos Aires: CLACSO. 2001. 359 pp.

Con un nuevo guiño hacia el conocido debate académico sobre los sistemas parlamentarios frente a los presidencialistas, el equipo coordinado por Jorge Lanzaro presenta un volumen que pretende no sólo reabrir aquellas discusiones sino enriquecerlas poniendo de manifiesto los avances experimentados por los regímenes presidencialistas en la región latinoamericana. Así, con la propuesta, nada trivial para los tiempos que corren, de intentar promocionar la reflexión teórica, llega este trabajo realizado íntegramente en la academia de América Latina y auspiciado por el programa académico del Consejo Latinoamericano de Ciencias Sociales (CLACSO) que compila estudios sobre Argentina, Bolivia, Brasil, Chile, México y Uruguay. Una prueba más de que desde su heterogeneidad se pueden analizar las cuestiones en común de estos regímenes presidenciales.

Desde la portada del libro en la que se recoge el saludo de los presidentes de Paraguay, Argentina, Brasil, Chile y Uruguay, en las deliberaciones de MERCOSUR en el 2000, al lector se le ofrece una imagen de unidad frente a las diferencias existentes. Unidad de régimen frente a las diferencias nacionales como se pondrá de manifiesto a lo largo de una obra que exceptuando los artículos generales que abren y cierran la edición, trata de capturar las singularidades de cada caso nacional seleccionado.

Jorge Lanzaro desde su «Tipos de presidencialismo y modos de gobierno en América Latina» inicia el volumen proponiendo una novedosa clasificación de carácter continuo sobre los regímenes presidencialistas. Incluso se atreve a calificar los analizados en la obra a los que los engloba dentro de la categoría de presidencialismo en transición que está convergiendo en la práctica en lo que se ha venido denominando como 
«presidencialismo de coalición». Una tipología que sin duda alguna invitará a la reflexión en todos los casos nacionales analizados.

Así el caso argentino inicia la serie de análisis nacionales desde el artículo «Los presidentes, equilibrios institucionales y coalición de gobierno en Argentina (19892000)» de Marcos Novaro donde se examina el balance de fuerzas desde la segunda presidencia de Menem con la formación de la Alianza y su estreno en el gobierno así como las debilidades y complicaciones a las que se ha enfrentado. Desde estas líneas el lector puede comprobar cómo casi adelantándose a los actuales acontecimientos, el autor se replantea con acierto el grado de consolidación y de eficacia del régimen presidencial argentino.

Desde el régimen especial boliviano de presidencialismo parlamentarizado, René Antonio Mayorga estudia las coaliciones lógicas que en un sistema de estas características se han venido formando desde 1989. Su tesis principal argumenta que dentro del contexto latinoamericano, la experiencia democrática boliviana se ha destacado por establecer mecanismos resolutorios a los problemas fundamentales de los regímenes presidencialistas principalmente a los impasses entre el poder ejecutivo y el legislativo, el inmovilismo institucional y particularmente los gobiernos minoritarios a través de pactos políticos interpartidistas. Esto ha conducido a la formación de gobiernos de coalición que se ha constituido como mecanismo fundamental de un sistema híbrido entre estructuras básicas presidencialistas con instrumentos parlamentarios.

Otro ejemplo de presidencialismo de coalición es que presentan Renato Lessa en su «Aventuras del Barón de Munchausen: notas sobre la tradición presidencialista brasileña» y Kurt E. von Mettenheim en «Presidencialismo, democracia y gobernabilidad en Brasil». Tanto desde una revisión histórica, ofrecida por Renato Lessa o limitándose a la década de 1990, como lo hace Kurt E. von Mettenheim, ambos autores abordan las prácticas de coalición en el sistema brasileño desde una visión mucho más prolongada debida a su práctica como clave de gobierno desde 1946 en respuesta a las «bases de la tradición republicana» de un país plural y heterogéneo.

Como en el caso anterior, también la experiencia chilena está referenciada por dos autores: Manuel Antonio Garretón en «La cuestión del régimen de gobierno en el Chile de hoy» y por Peter M. Siavelis en «Chile: las relaciones entre el poder ejecutivo y el poder legislativo después de Pinochet». Así, mediante el análisis del periodo postPinochet destacan cómo Chile retoma rasgos de las décadas anteriores a 1960 iniciado así un nuevo escenario de compromisos y coaliciones que cohabitan con enclaves autoritarios. Una democracia «limitada» en la que se alinean dos bloques políticos donde los partidos que se integran en cada uno conservan sus identidades.

Alonso Lujambio trata de hacer un análisis similar desde su «Adios a la excepcionalidad: régimen presidencial y gobierno dividido en México» donde explora las coaliciones de la última década en México desde la presidencia de Salinas y particularmente durante el Gobierno de Zedillo. Así a partir del marco conceptual elaborado por Linz para señalar los problemas de la democracia presidencial, pretende explicar los dilemas que enfrenta la política parlamentaria mexicana durante la legislatura 1997-2000. 
Con el análisis que realiza Jorge Lanzaro respecto al sistema uruguayo finalizan los casos nacionales de la obra. «Uruguay: las alternativas de un presidencialismo pluralista» analiza cómo los pactos «bipartidistas» en dicho país han confirmado un multipartidismo bipolar donde la política de bloques ha pasado a constituir su característica de identidad principal, que el autor califica como un tipo característico de presidencialismo pluralista.

Como colofón final de todos los debates analizados en este volumen el análisis de Daniel Chasquetti, en «Democracia, multipartidismo y coaliciones en América Latina: evaluando una difícil combinación» valora la forma en que los países de América Latina convivieron con la difícil combinación de presidencialismo y multipartidismo, demostrando cómo los presidencialismos multipartidistas gobernados por coaliciones mayoritarias son formatos político-institucionales eficaces para el mantenimiento de la estabilidad democrática mediante un análisis empírico del desempeño democrático de cincuenta y un gobiernos formados en doce países del continente durante el periodo 1978-2000.

Sin volver una vez más a reivindicar la conveniencia de un sistema presidencialista para el contexto latinoamericano, pero tampoco sin renunciar a las aportaciones críticas de una revisión de la problemática del presidencialismo en la región, esta obra aborda con maestría la problemática realizando una evaluación ponderada de la democracia presidencial desde un pertinente análisis comparado. Propone la categoría del «presidencialismo de coalición» como nueva y emergente dentro del ámbito latinoamericano, desafiando a quienes pensaban las coaliciones como exclusivas del parlamentarismo. Un desafío que adjetiva los sistemas presidenciales y que invita de una manera constructiva al debate académico. Frente a tantos interrogantes sobre la actual política latinoamericana, éste y todos los esfuerzos de reflexión son bienvenidos. Nosotros debemos estimularlos y por qué no empezar con esta obra.

ELENA MARTÍNEZ BARAHONA

Manuel Alcántara Sáez y Flavia Freidenberg (eds.). Partidos Políticos de América Latina. Salamanca: Ediciones Universidad de Salamanca, 2001. 2088 pp.

Diferentes autores clásicos ${ }^{1}$ fueron pioneros en el estudio de los partidos políticos como organizaciones internas; sin embargo, la escasez de este tipo de análisis frente al predominio de estudios acerca de otros aspectos de la organización -la financiación

1. Mosei Ostrogorski. Democracy and the Organization of Political Parties, vol. II. New York: Anchor Books, 1903; Robert Michels. Los partidos políticos. Un estudio sociológico de las tendencias oligárquicas de la democracia moderna. Buenos Aires: Amorrortu, 1911 (Quinta reimpresión, 1996) y Max WeBER. Economía y Sociedad. México: Fondo de Cultura Económica, 1922. 
electoral y no electoral, la disciplina interna, las familias de partidos, el tipo de incentivos utilizados para movilizar a los electores, las percepciones de las elites parlamentarias, la selección de candidatos y, en la última década, la publicación de estudios de caso- y la dificultad de llevar a cabo estudios comparativos entre partidos bien dentro del mismo sistema político bien entre sistemas políticos ${ }^{2}$ son las razones que justifican el porqué de esta investigación.

Se parte de una concepción doble de partido político como sistema: el partido político es un sistema político en sí mismo porque «tiene un proceso representativo, un sistema electoral, procedimientos para reclutar a líderes, definir objetivos y resolver conflictos internos» ${ }^{3}$ que, a su vez, forma parte del sistema político entendido como un todo. De la acción recíproca de ambas concepciones resultan cinco caras del partido: como organización burocrática, como organización voluntaria de miembros (ámbito interno), y como organización electoral, de gobierno y legislativa (ámbito externo).

Dos son los objetivos que persigue este trabajo: por una parte, el estudio de la estructura y funcionamiento de los partidos políticos latinoamericanos bajo la aplicación de un mismo esquema analítico, lo que permite la acumulación de información teórica y empírica comparada entre países de la región o entre partidos de un mismo sistema político. Por otra parte, enfatizar el papel de los partidos políticos como interlocutores imprescindibles entre el Estado y la sociedad.

De un total de dieciocho países latinoamericanos, agrupados en tres volúmenes -Cono Sur, Países Andinos y Centroamérica, México y República Dominicana-, se seleccionaron cincuenta y seis partidos conforme a los siguientes presupuestos: a) haber obtenido representación en la Cámara de Diputados en los tres últimos años; b) superar la barrera electoral del cinco por ciento durante el período indicado; c) contar con representación bien en todos los distritos electorales del país bien en aquellos donde su representación fuese significativa y d) que fuera un partido de especial importancia, sin el cual no pudiera entenderse la competición partidista.

Los capítulos mantienen una estructura homogénea: la introducción que describe el sistema institucional en relación con los partidos, así como las características principales de éstos; y el análisis de cada una de las caras de los partidos políticos arriba enunciadas. Sin embargo, es necesario subrayar los anexos de cada uno de los volúmenes, donde queda patente la perspectiva comparada del estudio, la sistematización de la recogida de datos, la localización de otras bases de datos de partidos políticos latinoamericanos, sus direcciones postales y páginas webs, y los rasgos que han guiado el proceso de elaboración de la obra: características originarias de las organizaciones partidistas, ubicación ideológica de los partidos en una escala de 0 (=izquierda) a 10

2. William SAlvo Crotty. A perspective for the comparative analysis of political parties. Comparative Political Studies. Londres: Sage Publications, pp. 267-295 y Richard KATZ y Peter MAIER. Three Faces of Party Organization: Adaptation and Change. Paper prepared for the XII World Congress of Sociology. Madrid, 9-13 july, 1990.

3. Véase Samuel Eldersveld. Political Parties. A Behavioral Analysis. Chicago: Rand Mc Nally, 1964, p. 1. 
(=derecha), características del funcionamiento interno de los partidos políticos, y características de la estructura interna de los partidos.

Este trabajo es fundamental no sólo por lo inédito tanto del material empírico como de su análisis comparado a nivel del conjunto de la región latinoamericana; sino que da cuenta de la necesidad de un enfoque integral en los estudios de las organizaciones partidistas si se quiere avanzar más allá del simple conocimiento de las estructuras y reglas que regulan la manera de relacionarse los individuos. Además, es una herramienta imprescindible, completa y útil para aquellos alumnos e investigadores que deseen conocer cómo funcionan «por dentro» los partidos latinoamericanos, al igual que establece la base para la formulación de nuevas preguntas de futuras investigaciones.

Por último, elogiar a sus editores el ingente esfuerzo humano que ha supuesto la coordinación de una obra de tal magnitud, y a sus colaboradores la profundidad, calidad, claridad y actualidad en la presentación de sus contenidos, tratamiento de los datos y conclusiones de una obra que ha sido el fruto de varios años dedicados a la investigación de los partidos políticos latinoamericanos dentro del proyecto «Partidos Políticos y Gobernabilidad en América Latina» financiado por la Comisión Interministerial de Ciencia y Tecnología del Ministerio de Educación y Cultura de España (Ref. Sec. 97-1458) dirigido por Manuel Alcántara Sáez y adscrito al Instituto Interuniversitario de Estudios de Iberoamérica y Portugal.

LEOPOLdo DíAz Moure

Álvaro Artiga-GonZÁLEz. La Política y los Sistemas de Partidos en Centroamérica. El Salvador: Fundaungo, 2000. 233 pp.

La literatura sobre los sistemas de partidos y el papel desempeñado por las organizaciones partidistas en los procesos de transición y durante la consolidación democrática ha sido abundante. Teniendo en cuenta que los procesos políticos a través de los cuales se fue gestando la aparición de los partidos (con la progresiva asunción de funciones) dentro de cada sistema político, se han desarrollado de forma diferente en cuanto al tiempo y modalidad, merece una especial atención averiguar si tal desarrollo ha tenido consecuencias para estos sistemas de partidos. Éste es uno de los objetivos planteados en La Política y los Sistemas de Partidos en Centroamérica.

Los acontecimientos acaecidos en la región centroamericana en la década de 1980 son el punto de partida para plantear la existencia de sistemas de partidos en Costa Rica, Honduras, Guatemala, El Salvador y Nicaragua, con rasgos diferenciados, a pesar de la tradicional convergencia que en términos económicos y sociales se presume de la región. 
Destaca especialmente la novedad de esta obra por ofrecer una comparación de área al explicar la génesis y configuración de los sistemas de partidos en la región centroamericana. En su argumentación, el autor distingue dos planos de observación, desde las elites y desde las masas. Álvaro Artiga adopta la primera opción al establecer una serie de variables políticas que pueden explicar la configuración de los sistemas de partidos, relacionadas con el comportamiento y estrategias de las principales elites de los sistemas políticos centroamericanos. En este sentido, el cuerpo central de su obra explora la relación del tipo de régimen político, la transición, el sistema electoral adoptado y el comportamiento de las elites sobre los sistemas de partidos en cada sistema político de la región.

La pregunta planteada por el autor hace referencia a la explicación de aspectos diferenciadores y semejantes en los actuales sistemas de partidos centroamericanos. Estos aspectos los agrupa en torno a tres binomios: estructuración/fluidez de la oferta partidista, bi/multipolaridad del sistema y moderación/polarización de la competencia. Considerando los sistemas de partidos como una institución fundamental para canalizar el conflicto y las demandas sociales, entre otras funciones, es preciso conocer si la estructuración actual obedece a las variables políticas señaladas.

El examen de los periodos previos a las transiciones políticas es el primer paso para demostrar la existencia de tales características en los sistemas de partidos antes de que se iniciara el proceso democratizador. Comienza de esta manera un recorrido histórico delimitando los periodos que básicamente tuvieron lugar en los cinco casos analizados, a partir del régimen oligárquico, si bien teniendo en cuenta los desfases temporales, la simultaneidad de ciertas etapas o los primeros rasgos diferenciadores en cada país centroamericano.

Es fundamental destacar que para cada uno de los binomios considerados y que constituyen las características del sistema de partidos, puede hallarse una relación con el grado de inclusión o exclusión de cada tipo de régimen establecido por las elites, lo cual concreta a su vez en un indicador doble: el grado en que el régimen permite a otros actores influir en el sistema y la oportunidad para la introducción de reformas que alteren la estructura de propiedad. De este análisis se constata que cuanto más incluyente se muestra el régimen considerado, más estructurado y moderado es el sistema de partidos y más claramente se articula el carácter bipolar, caso de Honduras y Costa Rica. Por el contrario, el carácter excluyente de los regímenes anteriores a la transición fue determinante para la aparición de competencia polarizada (en El Salvador, Guatemala y Nicaragua), como también lo fue el fracaso del reformismo, que se articuló en otro polo revolucionario (en Guatemala y El Salvador). Es especialmente relevante notar cómo el paso de una etapa a otra fue motivo de una renovación constante de la oferta partidista, cuya consecuencia más inmediata fue la fluidez de los sistemas de partidos, característica que aún perdura en El Salvador, Guatemala y Nicaragua.

La siguiente variable analizada es el cambio de régimen sobre los sistemas de partidos, una vez argumentada la presencia de características propias de aquéllos antes de la transición. Tomando como periodo clave la etapa 1979-1997 con la excepción 
de Costa Rica (y salvando la problemática de fijar el inicio /fin de la transición en los países centroamericanos), el autor advierte la presencia de continuidades en cuanto a la moderación/polarización y la multipolaridad de forma que el proceso democratizador vino a cristalizar aquellos rasgos presentes antes de la década de 1980.

El análisis de la configuración de los sistemas de partidos en Centroamérica se completa con el estudio de los sistemas electorales adoptados durante la transición y los efectos que éstos tuvieron para las formaciones políticas, si bien refuerzan una tendencia ya presente, con las características propias del sistema político considerado.

En un último momento, Artiga-González establece la relación entre el comportamiento de las elites (diferenciando las cúpulas partidistas de las elites parlamentarias) y el sistema de partidos. Donde tiene más relevancia las tendencias institucionales y del propio sistema (así como el papel de las masas) a la hora de analizar las estrategias de disputas o de división entre ellas.

La realidad observada muestra diferencias y similitudes entre los países, pese a contar todos ellos con características económico-sociales afines, lo cual viene a justificar la investigación, partiendo del marco de consolidación democrática presente a excepción de Costa Rica, en todos los sistemas políticos del área considerada.

A lo largo de la obra, el autor combina una estrategia diacrónica, por cuanto aborda la historia política de cada país, valiéndose de datos cualitativos, y sincrónica en la segunda parte de la obra, al realizar las comparaciones pertinentes entre los cinco casos centroamericanos en el modo de transición, el tipo de sistemas electorales o el comportamiento de las elites, capítulos en que el análisis cuantitativo tiene un mayor peso en su argumentación.

Finalmente, merece destacar el esfuerzo que realiza Artiga-González al abordar una comparación de área al explicar la génesis y desarrollo de los sistemas de partido en la región. Lo cual constituye un aporte fundamental en un contexto de consolidación democrática, ofreciendo al lector un análisis profundo de la naturaleza de los partidos en los sistemas políticos y con ello una mejor compresión de la situación actual de la política centroamericana.

Patricia Otero Felipe

Augusto BARRERa GuARDERAS. Acción colectiva y crisis política. El movimiento indí gena ecuatoriano en la década de los noventa. Quito: Abya Yala-Ciudad-OSAL, 2001.

El presente libro pretende analizar un fenómeno de rabiosa actualidad, a saber, interpretar la naturaleza y significado del emergente (y relevante) movimiento indigenista del Ecuador a la luz de las teorías de la acción colectiva y de los movimientos sociales. Así las cosas, no cabe duda que la obra reseñada tiene un notable interés, 
tanto por la ausencia de trabajos académicos existentes sobre el citado fenómeno como por la intención de interpretar la crítica (en términos políticos) década de los noventa en el Ecuador a través del acontecer de un colectivo históricamente ninguneado.

Para ello Augusto Barrera utiliza un esquema sumamente canónico. Destina los dos primeros capítulos del libro a reseñar esquemáticamente los diversos enfoques existentes sobre movimientos sociales y acción colectiva para pasar posteriormente, en el tercer capítulo, a describir someramente el acontecer político de Ecuador durante las décadas de los años setenta y ochenta. Posteriormente, en el capítulo cuarto expone la irrupción y significado del movimiento indígena, en el quinto describe su consolidación en la escena social y política del país y en el sexto muestra y detalla el rol hegemónico que jugó dicho autor en la crisis política que estalló en el último lustro. Finalmente, en el séptimo y último el autor nos desgrana, a modo de conclusión, sus 14 «tesis» sobre la evolución, consolidación y relevancia del indigenismo ecuatoriano organizado.

Elaborar un estudio de tal naturaleza no es nada fácil. En primer lugar debido a que la tarea de definir operativamente un «movimiento social» ya es complicada. Ciertamente, se podría decir que éste es un actor colectivo reconocido (con mayor o menor entusiasmo) en las arenas políticas de las poliarquías junto con otros actores más formalizados -como los partidos políticos, los grupos de presión e interés- con los que mantiene diferencias respecto al ámbito de incidencia política, la forma de participación, el discurso, la organización interna, las estrategias y la orientación. Pero por su rápida mutación y variedad de situaciones en que emergen los movimientos sociales existen muchas formas posibles de definir a este tipo de actor y su evolución. En este sentido, si bien el libro inicia con una reseña de las teorías que hacen referencia a este tipo de actores, mantiene cierta ambigüedad a la hora de definir la naturaleza del «movimiento indígena», al que a veces le atribuye características movimentistas y otras una invariable relación subordinada con su «interlocutor» institucionalizado (la CONAIE) o con la formación partidaria Pachakutik.

Con todo, cabe exponer también que si partimos de una definición operativa de movimiento social como «un actor político colectivo de carácter movilizador (y, por tanto, un espacio de participación) que persigue objetivos de cambio a través de acciones - generalmente no convencionales-, y que para ello, actúa con cierta continuidad, a través un alto nivel de integración simbólica y un bajo nivel de especificación de roles» supone afirmar que éstos son actores que inciden en todos los ámbitos de la política. En el ámbito simbólico porque es un sistema de narraciones al mismo tiempo que supone un haz de registros culturales, explicaciones y prescripciones de cómo determinados conflictos son expresados socialmente. En el ámbito interactivo porque es una forma específica de actor colectivo que está constituido por un conjunto de normas preestablecidas (provenientes de la sedimentación de una memoria y práctica histórica) que formal o informalmente constituyen una guía para la acción. En el ámbito institucional porque incide e impacta (transformándolos o poniéndolos en tensión) en los espacios que regulan y canalizan las conductas de los actores a través de acciones no convencionales -y generalmente disruptivas-. Y, finalemente, en el ámbito sustantivo porque (tal 
como se ha observado a lo largo de su desempeño) es un instrumento de cambio de la realidad.

A pesar de ello, en el presente libro, el autor focaliza su interés sólo en dos de los ámbitos expuestos, el interactivo y el institucional, dejando notables vacíos en los dos restantes. Así pues, son pocas las referencias que nos ofrece sobre la naturaleza del discurso y la simbología del movimiento en cuestión, y menos (aunque también es de más difícil prescripción) los rendimientos sustantivos producuidos por la emergencia del indigenismo en ese país andino.

Inequívocamente, el interés de la obra se focaliza en tres elementos que hacen referencia al ámbito de lo interactivo y de lo institucional. En primer lugar, el estudio de las microvariables que conllevan a los individuos pertenecientes a los colectivos indígenas de la Sierra a movilizarse alrededor de un movimiento que apela a las «identidades sociales básicas». En segundo lugar, el análisis de la estructura de oportunidades políticas que hacen posible que las conductas individuales de cientos de miles de personas y las estrategias políticas de unos cuantos líderes terminen por configurar uno de los movimientos indígenas con más visibilidad y protagonismo del subcontinente durante el último lustro. Y en tercer y último lugar, el estudio de la capacidad del movimiento en cuestión en penetrar en el andamiaje institucional del Estado ecuatoriano y, desde éste, poder incidir en su agenda política. Por ello todo ello, si bien el libro de Acción colectiva y crisis política tiene de algunos vacíos, es necesario felicitar el esfuerzo de analizar con un sofisticado utillaje académico una realidad que fácilmente se interpreta en clave política y, por ende, viciada por la pasión.

SALVADOR MARTÍ y PUIG 